\title{
Preoperative exercise training prevents functional decline after lung resection surgery: a randomized, single-blind controlled trial
}

\author{
Raquel Sebio García ${ }^{1}$, Maria Isabel Yáñez-Brage ${ }^{1}$, Esther Giménez Moolhuyzen ${ }^{2}$, Marta \\ Salorio Riobo ${ }^{3}$, Ana Lista Paz ${ }^{1}$ and Jose María Borro Mate ${ }^{2}$
}

${ }^{1}$ Physiotherapy Department, University of A Coruña, A Coruña, Spain

${ }^{2}$ Thoracic Department, University Hospital of A Coruña, A Coruña, Spain

${ }^{3}$ DC Fisioterapia Ares, A Coruña, Spain

\begin{abstract}
Objectives: To investigate the effects of a preoperative pulmonary rehabilitation programme in patients with lung cancer undergoing video-assisted thoracic surgery.

Design: Randomized, single-blind controlled trial.

Setting: Teaching hospital.

Subjects: Patients with suspected or confirmed lung cancer undergoing video-assisted thoracic surgery.

Intervention: Participants were randomized to either a prehabilitation group or a control group. Participants in the prehabilitation group underwent a combination of moderate endurance and resistance training plus breathing exercises three to five times per week.

Main measures: The primary outcome of the study was exercise capacity. Secondary outcomes were muscle strength (Senior Fitness Test), health-related quality of life (Short-Form 36) and the postoperative outcomes. Patients were evaluated at baseline (before randomization), presurgery (only the prehabilitation group), after surgery and three months post-operatively.

Results: A total of 40 patients were randomized and 22 finished the study (10 in the prehabilitation group and 12 in the control group). Three patients were lost to follow-up at three months. After the training, there was a statistically significant improvement in exercise tolerance $(+397$ seconds, $p=0.0001)$, the physical summary component of the SF-36 ( +4.4 points, $p=0.008)$ and muscle strength $(p<0.01)$. There were no significant differences between groups after surgery. However, three months postoperatively, significant differences were found in the mean change of exercise capacity $(\mathrm{p}=0.005)$, physical summary component $(p=0.001)$ and upper and lower body strength $(p=0.045$ and $\mathrm{p}=0.002$ )

Conclusions: A pulmonary rehabilitation programme before video-assisted thoracic surgery seems to improve patients' preoperative condition and may prevent functional decline after surgery.
\end{abstract}

\section{Keywords}

Cancer; Exercise programme; Preoperative care; Pulmonary rehabilitation; Quality of life 


\section{Introduction}

Lung resection surgery is regarded as the optimal treatment for non-small cell lung cancer, ${ }^{1}$ but considerable morbidity is associated especially among individuals with poor preoperative status. ${ }^{2}$ Newly diagnosed lung cancer patients are known to frequently exhibit poor cardiopulmonary fitness, low physical activity levels and an impaired health-related quality of life that can be further aggravated after lung resection surgery. ${ }^{3}$ Both the health-related quality of life and the preoperative cardiorespiratory fitness are two modifiable factors in the preoperative period that are strongly associated with postoperative mortality, morbidity and overall survival in patients with early non-small cell lung cancer. ${ }^{4,5}$ Preoperative pulmonary rehabilitation has been recommended as a preoptimization measure before thoracic surgery ${ }^{6}$ and preliminary studies have shown that pulmonary rehabilitation can improve exercise tolerance, functional capacity and pulmonary function. ${ }^{7,8}$ Furthermore, a recent systematic review and meta-analysis has found that preoperative exercise training can reduce hospital stay and postoperative complications in patients undergoing thoracotomy for non-small cell lung cancer. ${ }^{9}$ However, none of these studies have been conducted exclusively in patients undergoing minimally invasive procedures, which are known to ameliorate the impact associated with lung resection surgery and enhance postoperative recovery. Thus, with the advent of video-assisted thoracic surgery to resect lung tumours, there is an urge to determine whether or not pulmonary prehabilitation should be encouraged in this particular context.

Therefore, the aims of this study were: (1) to assess the effects of a preoperative pulmonary rehabilitation programme on exercise capacity, muscle strength and health-related quality of life in patients awaiting lung cancer surgery; and (2) to determine the effectiveness of the intervention in enhancing postoperative recovery after video-assisted thoracic surgery in comparison with the standard care.

\section{Materials and methods}

\section{Design}

This study was a randomized (1:1), assessor-blind controlled trial conducted at the University Hospital of A Coruña (Spain). The research protocol was designed according to the CONSORT guidelines (see CONSORT checklist) and was approved by the local Ethics Committee (CEIC Galicia, 2011/395). The protocol was registered in the database of clinical trials (clinicaltrials.gov) under the registration number NCT01963923.

\section{Participants}

From October 2013 to April 2015, lung cancer patients who were considered for lung resection surgery at the Thoracic Surgery Department of the University Hospital of A Coruña were assessed for eligibility. Inclusion and exclusion criteria are summarized in Table 1.

Table 1. Inclusion and exclusion criteria.

\begin{tabular}{ll}
\hline Inclusion criteria & Exclusion criteria \\
\hline - Adults $(\geqslant 18$ years old $)$ & $\begin{array}{l}\text { Neoadjuvant therapy with chemo- or radiotherapy in the six } \\
\text { months prior to surgery. }\end{array}$ \\
$\begin{array}{l}\text { - Suspected or confirmed diagnosis of NSCLC. } \\
\text { At least one of the following: (a) FEV } 1 \leqslant 80 \% \text { of predicted } \\
\text { value; (b) BMI } \geqslant 30 ;(c) \text { age } \geqslant 75 \text { years or }(\mathrm{d}) \text { two or more } \\
\text { co-morbidities identified in the Colinet Comorbidity Score. }\end{array}$ & $\begin{array}{l}\text { Inability to perform the exercise training. } \\
\text { Distance to the facility centre } \leqslant 80 \mathrm{~km} .\end{array}$
\end{tabular}


Potentially eligible patients were contacted by phone and then scheduled for an interview with a specialized physiotherapist. Those who agreed to participate gave written informed consent prior to any formal testing.

\section{Randomization and blinding}

Randomization was undertaken using a random-based computer programme (Epidat ${ }^{\circledR}$ v3.1 Xunta de Galicia, 2005) with an allocation ratio of 1:1. Individual allocations were placed in consecutively numbered and sealed opaque envelopes by a third person not involved in the study.

The baseline assessment was performed by the physiotherapist supervising the training programme; however, group allocation was only revealed to the physiotherapist after the initial evaluations were completed, ensuring the blindness of the assessment. Postoperative evaluations were performed by two independent physiotherapists who were specifically trained to perform the outcome measurements and who were unaware of the patients' allocation.

\section{Sample size estimation}

This study was powered to detect a difference in exercise capacity of 100 seconds between groups during the first postoperative evaluation (three weeks after surgery). This difference has been previously reported as the minimal clinically important difference for this outcome. ${ }^{10}$ To detect this difference with a probability of $\alpha=0.05$ and power $=80 \%$, using a bilateral hypothesis and estimating a $10 \%$ drop-out rate, 11 patients were required per group. Sample size calculation was performed using the Epidat ${ }^{\circledR}$ v3.1 Xunta de Galicia, 2005.

\section{Intervention}

Eligible patients were randomly allocated to the preoperative pulmonary rehabilitation programme (prehabilitation group) or to the standard care (control group). Participants in the control group received usual care (no exercise training), while the prehabilitation group engaged in a one-hour supervised pulmonary rehabilitation programme three to five times per week depending on the surgery date. The pulmonary rehabilitation programme took place in the rehabilitation room at the University Hospital of A Coruña and consisted of a combination of moderate endurance and resistance training according to the current guidelines. ${ }^{11}$ The intervention was supervised by a highly specialized physiotherapist with an expertise in respiratory physiotherapy and pulmonary rehabilitation.

Endurance training was performed using a calibrated cycle-ergometer (Monark 818 E, Monark Exercise $\mathrm{AB}$, Sweden) with an initial targeted duration of 30 minutes. Training load (Watts) was determined after a symptom-limited incremental cycle test performed according to the international guidelines. ${ }^{12}$ Patients were asked to discontinue the test owing to increased dyspnoea, muscle fatigue or any symptom of discomfort and the maximal workload maintained for at least 30 seconds was recorded as the peak work load $\left(\mathrm{W}_{\text {peak }}\right)$. Heart rate and oxygen saturation were continuously monitored during the test; blood pressure, rate of dyspnoea and leg fatigue were only recorded at the beginning and at the end of the test. For each training session patients were encouraged to perform 30 minutes of interval training (one minute at high intensity $\left(80 \%\right.$ of $\mathrm{W}_{\text {peak }}$ ) plus four minutes of active rest (performed at $50 \%$ of $\left.\mathrm{W}_{\text {peak }}\right)$ ). A five-minute warm-up and a four-minute cool down at $30 \%$ of the $\mathrm{W}_{\text {peak }}$ were also included in the 30minute time. At the beginning and at the end of the training, dyspnoea and leg fatigue were logged using the modified version of the Borg Scale. ${ }^{13}$ After the tenth session, intensity was re-examined during a new incremental cycle test to adjust the $\mathrm{W}_{\text {peak }}$ in order to maintain an optimal training stimuli.

Resistance training was performed using elastic bands (Thera-Band $\AA$, The Hygienic Corporation, Akron, Ohio, USA) and body-weight exercises. Four different bands of $1.25-\mathrm{m}$ long were used in this investigation, each one providing a different resistance. Training load was determined using a 25maximum repetition test, meaning that the patient could not perform more repetitions with the correct technique. ${ }^{14}$ Each training session patients performed six different exercises targeting the main muscle groups involved in daily life activities. The goal was to perform three sets of 15 repetitions for each exercise with a 45-second rest between sets. From the tenth onwards, the number of repetitions was 
maintained and the number of sets was increased to four if tolerated. Patients were encourage to maintain a moderate perceived rate of exhaustion according to the OMNI-Resistance Scale, which has been validated to be use with elastic bands. ${ }^{15}$

In addition, patients were asked to perform breathing exercises twice daily at home using a volumeoriented incentive spirometer (Coach 2 Incentive Spirometer® 22-4000 HD, Smith Medicals, USA). The protocol consisted of 30 sustained inspirations at $80 \%$ of the maximal Vital Capacity (measured previously with the same device) with an end-inspiratory hold of two to three seconds. Patients were instructed to perform six cycles of five repetitions each, with a one-minute rest between cycles.

\section{Outcomes}

The primary outcome for this study was exercise capacity measured with a Constant-load Cycle Endurance Test performed at $80 \%$ of the $\mathrm{W}_{\text {peak }}$. To perform the test, after two minutes of unloading pedalling, patients were instructed to exercise for as long as possible until maximal exhaustion was achieved or the patient was unable to maintain the minimal pedalling rate. If exercise endurance exceeded more than 15 minutes the test was terminated. ${ }^{16}$ Dyspnoea and leg fatigue were recorded at the beginning and at the end of the test. Heart rate and oxygen saturation were recorded every minute.

Secondary outcomes included functional capacity, muscle strength and the health-related quality of life. Functional capacity was assessed with a 6-minute walk test. The test was performed according to the international recommendations. ${ }^{17}$ Duplicate tests were completed at baseline for reliability and the maximum distance was used for the analysis. The values achieved during the test were compared against the Spanish reference equations. ${ }^{18}$

Upper and lower body strength were also measured using two items included in the Senior Fitness Test initially designed by Rikli and Jones: ${ }^{19}$ the Arm Curl Test and the 30's Chair-to-Stand Test. Both tests were adapted to the metric measures according to authors' recommendations.

Health-related quality of life was assessed using the second version of the Short-Form 36 Health Survey (SF-36v2) and the results were compared against the Spanish reference values. ${ }^{18}$

Postoperative outcomes, including length of hospital stay, and the incidence of postoperative pulmonary complications were also recorded. Postoperative pulmonary complications were classified according to the Melbourne Group Scale, which was specifically designed to identify those complications that were more likely to be prevented with a physiotherapy intervention. ${ }^{20}$

Finally, medical and relevant sociodemographic data including age, gender, weight, height, history of smoking, the Colinet Co-morbidity Score (CCS), ${ }^{21}$ symptomatology and pulmonary function tests were collected from the medical charts.

Patients were evaluated three times during the study period: At baseline, after hospital discharge and three months post-operatively. Patients in the prehabilitation group were also evaluated immediately after the programme and before surgery.

\section{Statistical analysis}

A descriptive analysis of the main variables in the study was conducted for each group prior to further analyses. Distribution was assessed using the Kolmogorov-Smirnov Test for each quantitative variable in each group. Changes in the primary and secondary variables pre- to postintervention in the prehabilitation group were assessed using a pair $t$-test (variables normally distributed). Inter-group comparisons for the primary and secondary outcomes (exercise capacity, muscle strength and health-related quality of life domains) were performed using independent $t$-tests. Comparison of length of hospital stay between groups was performed using a Mann-Whitney test. The incidence of postoperative complications between groups was assessed using a chi square test.

All statistical analyses were calculated using the SPSS package for Windows (Version 22, IBM Corporation, Chicago IL, USA) and a $p$ value of $<0.05$ was considered statistically significant. 


\section{Results}

During the 18-month recruitment period, 319 patients were scheduled for lung resection surgery for suspected or confirmed lung cancer at our hospital. Of those, 68 potentially eligible patients (21.3\%) were contacted and $46(67.6 \%)$ signed the informed consent. Six patients (13\%) withdrew before randomization, therefore 40 patients were randomized and $22(55 \%)$ completed at least one postoperative evaluation and were analysed (10 patients in the prehabilitation group and 12 in the control group). At three months, two patients in the control group and one in the prehabilitation group were lost to followup. A flow diagram of the study selection is shown in Figure 1. The study was closed according to the pre-approved funding schedule.

The baseline characteristics of the participants included in the study are shown in Table 2. Despite the randomization, patients in the prehabilitation group tended to have a higher body mass index, lower pulmonary function and lower peak exercise capacity.

Mean time (SD) from baseline assessment to surgery was 54.5 days (15.4) in the prehabilitation group and $53.5(20)$ in the control group $(p=0.899)$. Patients in the prehabilitation group attended a median of 16 sessions (range 8-25) with no adverse events being reported. Median time from surgery to the first and second evaluation for both groups were 3.5 weeks (interquartile range: 1.5-4.9) and 14 weeks (interquartile range: 13.1-15.7), respectively. No significant differences were observed between groups ( $p=0.093$ and $p=0.842$ ). All patients underwent video-assisted thoracic surgery through one or two ports performed by highly experienced surgeons. The operation report was checked after the surgery to ensure that the surgery was performed with the same technique. There was only one reconversion to thoracotomy (control group) and the patient was excluded from the analysis.

Results of the preoperative pulmonary rehabilitation programme in the prehabilitation group are shown in Table 3, while the inter-group comparison can be found in Table 4. Although no significant differences between groups were observed at three weeks in any of the variables analysed, three months postoperatively there were statistically significant differences in the mean change for the exercise capacity, the physical component summary and the upper and lower muscle strength assessment leading to two opposite trends in patients' recovery: While patients in the prehabilitation group were able to maintain and/or increase their baseline values in all the parameters examined, patients in the control group exhibited a progressive decline throughout the follow-up, especially in exercise capacity.

Finally, no significant differences were found in the postoperative outcomes between groups. Hospital stay was similar for both groups (two vs. three median days in the prehabilitation and control group respectively; $p=0.539$ ). Any patient was diagnosed with a postoperative pulmonary complication according to the Melbourne Group Scale although 50\% $(n=5)$ of the patients in the prehabilitation group and 66\% $(n=8)$ in the control group scored positively in at least one of the items of the scale $(p=0.361)$. 


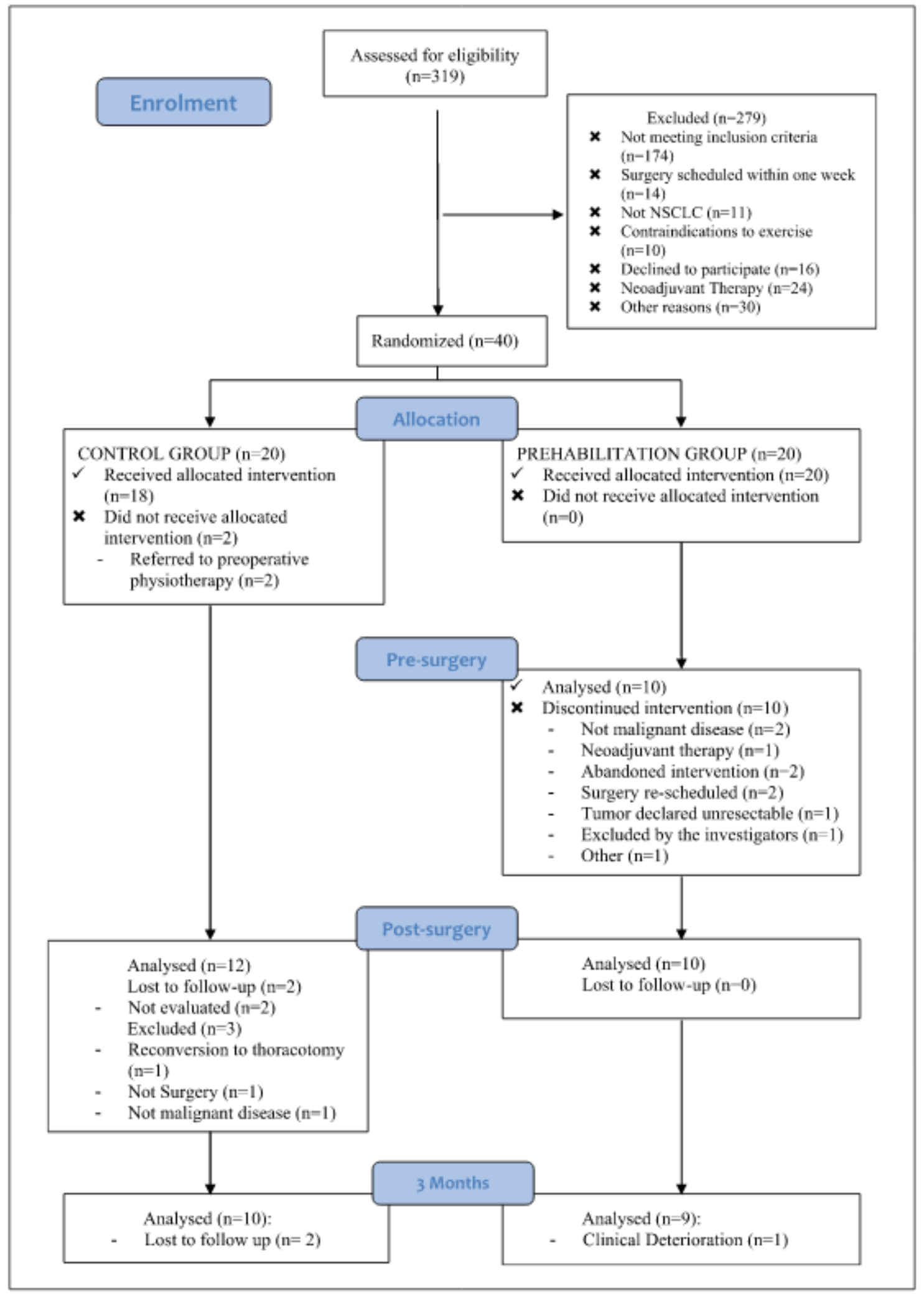

Figure 1. Flow diagram of the study NSCLC: non-small cell lung cancer. 
Table 2. Baseline characteristics of the patients included in the study.

\begin{tabular}{|c|c|c|}
\hline \multirow[t]{2}{*}{ Variable } & \multicolumn{2}{|l|}{ Group } \\
\hline & Control $(n=12)$ & Rehabilitation $(n=10)$ \\
\hline Age & $69.4 \pm 9.4$ & $70.9 \pm 6.1$ \\
\hline Sex (male/female) & $11 / 1$ & $9 / 1$ \\
\hline BMI & $25.7 \pm 2.8$ & $29.4 \pm 4.3$ \\
\hline \multicolumn{3}{|l|}{ History of smoking $(n, \%)$} \\
\hline No & $2(16.7 \%)$ & $2(20 \%)$ \\
\hline Former & $7(58.3 \%)$ & $8(80 \%)$ \\
\hline Current & $3(25 \%)$ & 0 \\
\hline Package/year & $39.5 \pm 32.1$ & $50.7 \pm 34.8$ \\
\hline $\mathrm{CCS}$ & $8.7 \pm 4.2$ & $9.3 \pm 4.9$ \\
\hline Respiratory disease $(n, \%)$ & $4(33.3 \%)$ & $7(70 \%)$ \\
\hline Cardiovascular disease $(n, \%)$ & $9(75 \%)$ & $8(80 \%)$ \\
\hline Renal impairment $(n, \%)$ & 0 & 0 \\
\hline Diabetes mellitus $(n, \%)$ & $3(25 \%)$ & $1(10 \%)$ \\
\hline $\operatorname{Alcoholism}(n, \%)$ & 0 & 0 \\
\hline PHx cancer $(n, \%)$ & $6(50 \%)$ & $4(40 \%)$ \\
\hline Symptomatology $(n, \%)$ & $8(66.7 \%)$ & $10(100 \%)$ \\
\hline Cough & $3(25 \%)$ & $3(30 \%)$ \\
\hline Dyspnoea & $3(25 \%)$ & $7(70 \%)$ \\
\hline Expectoration & $7(58.3 \%)$ & $4(40 \%)$ \\
\hline Other & $4(33.3 \%)$ & $2(20 \%)$ \\
\hline $\mathrm{FEV}_{1}(\%$ of predicted $)$ & $87.6 \pm 26.1$ & $69.2 \pm 15.1$ \\
\hline FVC (\% of predicted) & $80.1 \pm 18$ & $71.2 \pm 15$ \\
\hline $\mathrm{FEV}_{1} / \mathrm{FVC}$ & $68.8 \pm 6$ & $61.2 \pm 9.4$ \\
\hline 6MWT (metres) & $507.7 \pm 9$ & $420.2 \pm 116.3$ \\
\hline $6 \mathrm{MWT}$ ( $\%$ of predicted) & $79.8 \pm 11.4$ & $70.1 \pm 18.2$ \\
\hline Maximal workload (Watts) (median, IQR) & $75(67.5-84.4)$ & $67.5(50-75)$ \\
\hline Maximal workload ( $\%$ of predicted) & $55.4 \pm 16.7$ & $51.35 \pm 15.18$ \\
\hline CCET (seconds) & $366.83 \pm 205$ & $322.40 \pm 96$ \\
\hline Curl arm (no. repetitions) & $17.3 \pm 3.5$ & $13.4 \pm 3$ \\
\hline Sit to stand (no. repetitions) & $12.7 \pm 2.5$ & $11.5 \pm 3.7$ \\
\hline
\end{tabular}

6MWT: 6-minute walk test; BMI: body mass index; CCET: constant-load cycle endurance test; CCS: Colinet Co-morbidity Score; $\mathrm{FEV}_{1}$ : forced expiratory volume in 1 second; FVC: forced vital capacity; IQR: interquartile range; PHx: past history; SBP: systolic blood pressure.

Values are expressed in mean \pm standard deviation unless otherwise indicated.

Table 3. Changes in main outcomes pre- to postintervention (before surgery) in the prehabilitation group.

\begin{tabular}{|c|c|c|c|c|c|c|}
\hline \multirow[t]{2}{*}{ Variable } & \multirow[t]{2}{*}{$\mathrm{n}$} & \multirow[t]{2}{*}{$\begin{array}{l}\text { Baseline } \\
\text { (mean, SD) }\end{array}$} & \multirow[t]{2}{*}{ Mean change } & \multicolumn{2}{|l|}{ CI $(95 \%)$} & \multirow[t]{2}{*}{$p$ value $^{\mathrm{a}}$} \\
\hline & & & & Lower limit & Upper limit & \\
\hline CCET (seconds) & 10 & $322.4(96.2)$ & +396.6 (197.9) & +255 & +538.2 & $<0.001$ \\
\hline Arm curl (no. repetitions) & 10 & $13.40(3)$ & $+2.9(2.1)$ & +1.4 & +4.4 & 0.002 \\
\hline Chair-to-stand (no. repetitions) & 10 & $11.5(3.7)$ & $+0.9(1.2)$ & +0.4 & +1.7 & 0.041 \\
\hline Bodily pain & 10 & $64.5(26.3)$ & $+4.7(22.1)$ & -11.1 & +20.5 & 0.518 \\
\hline General health & 10 & $41.9(20.2)$ & $+10.3(16)$ & -1.1 & +21.7 & 0.072 \\
\hline Vitality & 10 & $52(16.5)$ & $+3(14.4)$ & -7.3 & +13.3 & 0.526 \\
\hline Social functioning & 10 & $87.5(22)$ & $+10(24.1)$ & -7.3 & +27.3 & 0.223 \\
\hline Role emotional & 10 & $60.6(19.2)$ & $+12(20.6)$ & -2.7 & +26.7 & 0.098 \\
\hline
\end{tabular}

${ }^{1}$ SD: standard deviation; CCET: Constant Cycle Endurance Test; CI: confidence interval; PCS: physical component summary; MCS: mental component summary.

${ }^{\mathrm{a}}$ Paired $t$-test analysis. 
Table 4. Changes in exercise capacity, functional capacity, muscle strength and health-related quality of life during the study period in both groups.

\begin{tabular}{|c|c|c|c|c|c|c|c|}
\hline Variable & Group $(n)$ & Baseline & $\begin{array}{l}\text { Postsurgery } \\
\text { (MD) }\end{array}$ & $\mathrm{p}$ value ${ }^{*}$ & Group (n) & $\begin{array}{l}3 \text { months } \\
\text { (MD) }\end{array}$ & $p$ value \\
\hline \multirow[t]{2}{*}{ CCET (seconds) } & $\begin{array}{l}\text { Prehabilitation } \\
(n=10)\end{array}$ & $\begin{array}{c}322.4 \pm \\
96.2\end{array}$ & $+137.7 \pm 268.2$ & 0.097 & $\begin{array}{l}\text { Prehabilitation } \\
(n=9)\end{array}$ & $\begin{array}{l}+226 \pm \\
269.4^{\mathrm{a}}\end{array}$ & 0.005 \\
\hline & $\begin{array}{l}\text { Control }(n= \\
12)\end{array}$ & $366.8 \pm 205$ & $-25.8 \pm 16.71$ & & Control $(n=10)$ & $\begin{array}{c}-137.8 \pm \\
221.7\end{array}$ & \\
\hline \multirow[t]{2}{*}{ 6MWT (metres) } & $\begin{array}{l}\text { Prehabilitation } \\
(n=10)\end{array}$ & $\begin{array}{c}420.11 \pm \\
116.3\end{array}$ & $\begin{array}{c}-15.55 \pm \\
47.731\end{array}$ & 0.500 & $\begin{array}{l}\text { Prehabilitation } \\
(n=9)\end{array}$ & $1.88 \pm 34.7$ & 0.186 \\
\hline & $\begin{array}{l}\text { Control }(n= \\
12)\end{array}$ & $\begin{array}{c}514.5 \pm \\
100.9\end{array}$ & $-27.7 \pm 33.7^{*}$ & & Control $(n=10)$ & $\begin{array}{c}-31.5 \pm \\
64.6\end{array}$ & \\
\hline \multirow[t]{2}{*}{$\begin{array}{l}\text { Arm -Curl (no. } \\
\text { repetitions) }\end{array}$} & $\begin{array}{l}\text { Prehabilitation } \\
(n=10)\end{array}$ & $13.4 \pm 3$ & $+1.9 \pm 3$ & 0.105 & $\begin{array}{l}\text { Prehabilitation } \\
(n=9)\end{array}$ & $+1.8 \pm 3.3$ & 0.045 \\
\hline & $\begin{array}{l}\text { Control }(n= \\
12)\end{array}$ & $17.3 \pm 3.5$ & $-0.25 \pm 2.9$ & & Control $(n=10)$ & $-1.8 \pm 3.5$ & \\
\hline \multirow[t]{2}{*}{$\begin{array}{l}\text { Chair-to-Stand } \\
\text { (no. repetitions) }\end{array}$} & $\begin{array}{l}\text { Prehabilitation } \\
(n=10)\end{array}$ & $11.5 \pm 3.7$ & $-0.55 \pm 3.5$ & 0.531 & $\begin{array}{l}\text { Prehabilitation } \\
(n=9)\end{array}$ & $+2 \pm 2.2^{*}$ & 0.002 \\
\hline & $\begin{array}{l}\text { Control }(n= \\
12)\end{array}$ & $12.7 \pm 2.5$ & $+0.5 \pm 3.9$ & & Control $(n=10)$ & $-1.3 \pm 1.8 *$ & \\
\hline \multirow[t]{2}{*}{ PCS } & $\begin{array}{l}\text { Prehabilitation } \\
(n=10)\end{array}$ & $40.8 \pm 8$ & $-2.8 \pm 5.8$ & 0.067 & $\begin{array}{l}\text { Prehabilitation } \\
(n=9)\end{array}$ & $+4.3 \pm 4^{*}$ & 0.001 \\
\hline & $\begin{array}{l}\text { Control }(n= \\
12)\end{array}$ & $49.48 \pm 5.5$ & $-7.4 \pm 5.3^{*}$ & & Control $(n=10)$ & $-4.8 \pm 5.8^{*}$ & \\
\hline
\end{tabular}

MD: mean difference; CCET: Constant Cycle Endurance Test; 6MWT: 6-minute walk test; PCS: physical component summary. ${ }^{\mathrm{a}}$ Independent $t$-test analysis

\section{Discussion}

The results found in our study suggest that a preoperative exercise-based programme before lung resection surgery can effectively improve patients' preoperative status by increasing exercise capacity, muscle strength and the physical component of the health-related quality of life. More importantly, in comparison with the standard care, it seems that preoperative exercise training can also minimize the physical deconditioning associated with lung resection and accelerate functional recovery after videoassisted thoracic surgery. However, this was a small study with a high number of drop-outs thus our findings must be interpreted carefully. Nevertheless, they are proof of the principle that preoperative pulmonary rehabilitation before video-assisted thoracic surgery may play an important role in preventing functional decline after surgery similarly to what has been observed after open thoracotomies.

Pulmonary rehabilitation has been recently acknowledged as a cornerstone in the management of patients with lung cancer. ${ }^{22}$ To date, several studies have consistently shown that preoperative exercise training can increase exercise capacity, ${ }^{8,23-26}$ functional capacity ${ }^{7,25-27}$ and pulmonary function ${ }^{7,24,25,27}$ in patients with lung cancer awaiting lung resection surgery both pre to postintervention and comparing with a control group. ${ }^{7,28}$ In our study, we also found a significant and clinically meaningful improvement in exercise capacity (endurance time) of 369.6 seconds (197) pre- to posttraining, which corresponds to a $123 \%$ increase compared with baseline. More importantly, patients were able to maintain this improvement after the surgery throughout the first three months, which contrasts with the control group who showed a progressive decline over the study period. In a similar investigation, Coats et al. ${ }^{26}$ also found a $60 \%$ improvement in exercise capacity after a four-week home-based training programme in patients awaiting video-assisted thoracic surgery. Interestingly, in this investigation, the authors did not report any significant change in the maximum oxygen uptake after the training suggesting that incremental exercise tests may not be the more responsive to detect exercise-related changes.

In addition to exercise capacity, we also found a significant improvement both in upper and lower muscle strength after the training. In a previous pilot study, our group already showed that elastic bands were an effective way to improve muscle strength in patients with lung cancer. ${ }^{29}$ Although resistance training is more commonly delivered using free weights or weight machines, the use of low-tech equipment is gaining more popularity. Several studies have found that elastic bands are as effective as traditional equipment to improve muscle strength in sedentary older adults ${ }^{30}$ and those with chronic respiratory diseases, ${ }^{31}$ including patients with lung cancer. ${ }^{32}$ The results obtained in this randomized 
controlled trial are in line with these previous investigations and further reinforce that low-cost training is a valid alternative for improving muscle strength in this population.

On the other hand, the effects of exercise training in health-related quality of life appear less clear. Previous systematic reviews conducted among patients with lung cancer have highlighted the lack of consistency among studies regarding the effectiveness of exercise training on this outcome. ${ }^{9,33}$ The results of this randomized controlled trial, however, indicate that a preoperative intervention focused on endurance and strength training can significantly improve the physical role and the physical component summary prior to lung resection surgery. This finding is of great interest given that the preoperative physical component summary is associated with overall and cancer-specific survival in patients with stage I Non-Small Cell Lung Cancer. ${ }^{5}$ More importantly, the increase in the physical component summary was also sustained throughout the first three months after the surgery in comparison with the control group who showed the opposite tendency.

Finally, preoperative exercise training has shown the potential of enhancing postoperative recovery and reduce in-hospital costs in patients undergoing cardiothoracic and abdominal surgery. ${ }^{9,34}$ Particularly, in patients undergoing lung resection surgery, our group found that there was a reduction of almost five days in hospital stay (mean difference $=4.83$ days, 95\% CI: 3.8 to 5.9) and a $55 \%$ reduction in the relative risk for post-operative complications in those patients undergoing preoperative exercise training. ${ }^{9}$ However, in this study, we could not find any significant difference between groups neither in hospital stay nor in the postoperative complications. This finding can be explained by several factors including the experience of the thoracic surgery team plus the low-risk profile of the patients included in our study.

Our study has several limitations that must be acknowledged. First of all, the study included patients with suspected (clinical) or confirmed lung cancer (histological biopsy), which resulted in some patients being diagnosed with lung metastases or benign disease. Despite randomization, patients in the prehabilitation group had poorer pulmonary function and a higher body mass index, which resulted in a higher percentage of them undergoing a sub-lobar resection than those in the control group. However, the differences were not statistically significant $(p=0.229)$ and a univariate general linear model showed no interaction between the exercise capacity and the extent of the resection (data not shown). It is also important to acknowledge the high number of drop-outs in this study and the potential implications. Of the 20 patients randomized to the prehabilitation group, only $50 \%$ completed the study, which lead us to perform a per-protocol analysis instead of an intention-to-treat analysis owing to the large percentage of patients with relevant missing data. On the bright side, this low completion rate had little to do with the preoperative pulmonary rehabilitation programme, which was well tolerated and related to no adverse events.

\section{Clinical messages}

- Our results suggest that a preoperative exercise-based programme can improve exercise capacity, muscle strength and the physical component of the health-related quality of life in patients with lung cancer.

- In these patients, preoperative exercise training could play an important role in preventing functional decline after surgery enhancing postoperative recovery.

\section{Acknowledgements}

The authors of this manuscript would like to thank the Thoracic Ward staff at the University Hospital of A Coruña for their contribution to this investigation, as well as the Physiotherapy Department staff at the University of A Coruña, especially Dr Sonia Souto Camba. 


\section{Authorship}

Dr Raquel Sebio was the main investigator of this study. She designed the intervention, supervised the exercise programme, analysed the data and wrote the manuscript. Professor MI Yáñez and Dr Borro were also responsible for the design of the intervention and manuscript editing. Miss Esther Giménez and Miss Marta Salorio were the blind therapists in charge of the postoperative evaluations. Finally, Dr Ana Lista helped with the data collection and data analysis. All authors reviewed the final version of the manuscript.

\section{Conflict of interest}

The author(s) declared no potential conflicts of interest with respect to the research, authorship, and/or publication of this article.

\section{Funding}

The author(s) disclosed receipt of the following financial support for the research, authorship, and/or publication of this article: Miss Raquel Sebio was economically supported by a three-year predoctoral research fellowship from the Xunta de Galicia when conducting this clinical trial.

\section{References}

1. Howington JA, Blum MG, Chang AC, Balekian AA, Murthy SC. Treatment of stage I and II non-small cell lung cancer: Diagnosis and management of lung cancer, 3rd ed. American College of Chest Physicians evidence-based clinical practice guidelines. Chest 2013; 143(5 Suppl): e278S-313S.

2. Benzo R, Kelley GA, Recchi L, Hofman A, Sciurba F. Complications of lung resection and exercise capacity: A meta-analysis. Respir Med 2007; 101(8): 1790-1797.

3. Granger CL, McDonald CF, Irving L, . Low physical activity levels and functional decline in individuals with lung cancer. Lung Cancer 2014; 83(2): 292-299.

4. Jones LW, Watson D, Herndon JE2nd, . Peak oxygen consumption and long-term allcause mortality in nonsmall cell lung cancer. Cancer 2010; 116(20): 4825-4832.

5. Pompili C, Salati M, Refai M, . Preoperative quality of life predicts survival following pulmonary resection in stage I non-small-cell lung cancer. Eur J Cardiothorac Surg 2013; 43(5): 905-910.

6. Jones NL, Edmonds L, Ghosh S, Klein AA. A review of enhanced recovery for thoracic anaesthesia and surgery. Anaesthesia 2013; 68(2): 179-189.

7. Morano MT, Araújo AS, Nascimento FB, . Preoperative pulmonary rehabilitation versus chest physical therapy in patients undergoing lung cancer resection: A pilot randomized controlled trial. Arch Phys Med Rehab 2013; 94(1): 53-58.

8. Jones LW, Peddle CJ, Eves ND, . Effects of presurgical exercise training on cardiorespiratory fitness among patients undergoing thoracic surgery for malignant lung lesions. Cancer 2007; 110(3): 590-598.

9. Sebio Garcia R, Yanez Brage MI, Gimenez Moolhuyzen E, Granger CL, Denehy L. Functional and postoperative outcomes after preoperative exercise training in patients with lung cancer: A systematic review and meta-analysis. Interact Cardiovasc Thorac Surg 2016; 23(3): 486-497

10. Laviolette L, Bourbeau J, Bernard S, . Assessing the impact of pulmonary rehabilitation on functional status in COPD. Thorax 2008; 63(2): 115-121.

11. Spruit MA, Singh SJ, Garvey C, . An official American Thoracic Society/European Respiratory Society statement: Key concepts and advances in pulmonary rehabilitation. Am J Respir Crit Care Med 2013; 188(8): e13-64.

12. ATS/ACCP. ATS/ACCP Statement on Cardiopulmonary Exercise Testing. Am J Respir Crit Care Med 2003; 167: 211-277.

13. Borg G. Ratings of perceived exertion and heart rates during short-term cycle exercise and their use in a new cycling strength test. Int J Sports Med 1982; 3(3): 153-158.

14. Newsam C. Intratester reliability for determining an 8-repetition maximum for 3 shoulder exercises using elastic bands. J Sport Rehabil 2005; 14(1): 35.

15. Colado JC, Garcia-Masso X, Triplett TN, Flandez J, Borreani S, Tella V. Concurrent validation of the OMNI-resistance exercise scale of perceived exertion with Thera-band resistance bands. J S Cond Res 2012; 26(11): 3018-3024. 
16. Van 't Hul A, Gosselink R, Kwakkel G. Constant-load cycle endurance performance: Test-retest reliability and validity in patients with COPD. J Cardiopulm Rehabil 2003; 23(2): 143-150.

17. American Thoracic Society. ATS statement: Guidelines for the six-minute walk test. Am J Respir Crit Care 2002; 166(1): 111-117.

18. Gimeno-Santos E, Arbillaga-Etxarri A, Vilaró J, . Reference equations for 6-minute walk test in Spanish population. Eur Respi J 2015; 46(suppl 59): PA1543. DOI: 10.1183/13993003.congress-2015.PA1543.

19. Rikli RE, Jones CJ. Development and validation of criterion-referenced clinically relevant fitness standards for maintaining physical independence in later years. The Gerontologist. 2013; 53(2): 255-267.

20. Reeve JC, Nicol K, Stiller K, McPherson KM, Denehy L. Does physiotherapy reduce the incidence of postoperative complications in patients following pulmonary resection via thoracotomy? A protocol for a randomised controlled trial. J Cardiothorac Surg 2008; 3: 48.

21. Colinet B, Jacot W, Bertrand D, . A new simplified comorbidity score as a prognostic factor in non-small-cell lung cancer patients: Description and comparison with the Charlson's index. Br J Cancer 2005; 93(10): 1098-1105.

22. Wang H, Liu X, Rice SJ, Belani CP. Pulmonary rehabilitation in lung cancer. PM R 2016; 8(10): 990-996.

23. Bobbio A, Chetta A, Ampollini L, . Preoperative pulmonary rehabilitation in patients undergoing lung resection for non-small cell lung cancer. Eur J Cardiothorac Surg 2008; 33(1): 95-98.

24. Bagan P, Oltean V, Ben Abdesselam A, Couffinhal JC, De Cremoux H. Pulmonary rehabilitation and prophylactic non-invasive ventilation before lung cancer surgery in very high-risk patients. Rev Mal Respir 2013; 13: S30-S31.

25. Divisi D, Di Francesco C, Di Leonardo G, Crisci R. Preoperative pulmonary rehabilitation in patients with lung cancer and chronic obstructive pulmonary disease. Eur J Cardiothorac Surg 2013; 43(2): 293-296.

26. Coats V MF, Simard S, Frechette E, . Feasibility and effectiveness of a home-based exercise training program before lung resection surgery. Can Respir J 2013; 20(2): e1016. Google Scholar CrossRef, Medline

27. Cesario A, Ferri L, Galetta D, . Pre-operative pulmonary rehabilitation and surgery for lung cancer. Lung Cancer 2007; 57(1): 118-119.

28. Stefanelli F, Meoli I, Cobuccio R, . High-intensity training and cardiopulmonary exercise testing in patients with chronic obstructive pulmonary disease and non-smallcell lung cancer undergoing lobectomy. Eur J Cardiothorac Surg 2013; 44(4): e260265.

29. Sebio R, Yáñez-Brage MI, Giménez-Moolhuyzen E, Valenza MC, Reychler G, Cahalin LP. Impact of a pre-operative pulmonary rehabilitation program on functional performance in patients undergoing video-assisted thoracic surgery for lung cancer. Arch Bronconeumol 2016; 52(5): 231-232.

30. Lubans DR, Mundey CM, Lubans NJ, Lonsdale CC. Pilot randomized controlled trial: Elastic-resistance-training and lifestyle-activity intervention for sedentary older adults. $\mathrm{J}$ Aging Phys Act 2013; 21(1): 20-32.

31. Murphy N, Bell C, Costello RW. Extending a home from hospital care programme for COPD exacerbations to include pulmonary rehabilitation. Respir Med 2005; 99(10): 1297-1302.

32. Peddle-McIntyre CJ, Bell G, Fenton D, McCargar L, Courneya KS. Feasibility and preliminary efficacy of progressive resistance exercise training in lung cancer survivors. Lung cancer 2012; 75(1): 126-132.

33. Pouwels S, Fiddelaers J, Teijink JAW, Woorst JFt, Siebenga J, Smeenk FWJM. Preoperative exercise therapy in lung surgery patients: A systematic review. Respir Med 2015; 109(12): 1495-1504.

34. Valkenet K, van de Port IG, Dronkers JJ, de Vries WR, Lindeman E, Backx FJ. The effects of preoperative exercise therapy on postoperative outcome: A systematic review. Clin Rehabil 2011; 25(2): 99-111. 